\title{
The Computer-Aided Simulation of Deformation and Fracture of Water-Saturated Elastic Porous Material with Hybrid Cellular Automaton Method
}

\author{
Andrey V. Dimaki ${ }^{1,2, a)}$, Evgeny V. Shilko ${ }^{1,2, ~ b)}$, Sergey V. Astafurov ${ }^{1,2, c)}$, \\ and Sergey G. Psakhie ${ }^{1,3, \mathrm{~d})}$ \\ ${ }^{1}$ Institute of Strength Physics and Materials Science SB RAS, Tomsk, 634055, Russia \\ ${ }^{2}$ National Research Tomsk State University, Tomsk, 634050, Russia \\ ${ }^{3}$ National Research Tomsk Polytechnic University, Tomsk, 634050, Russia

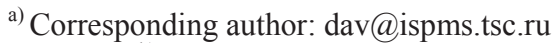 \\ b) shilko@ispms.tsc.ru \\ c)svastafurov@gmail.com \\ d) sp@ispms.tsc.ru
}

\begin{abstract}
A method of numerical simulation of liquid-saturated porous media, that represents a combination of particle method and finite-difference method, namely, the hybrid cellular automaton method was proposed. It allows taking into account inelastic deformations, dilation and fracture of solid skeleton as well as the influence of pore pressure on the stress state of the skeleton and the redistribution of a liquid in filtration volume of porous medium. The method was applied to study the influence of viscous compressible liquid in pores of material on its strength and fracture. It has been shown that the strength of brittle liquid-saturated specimens depends on the material properties and the geometry of porosity, the physical-mechanical properties of the liquid etc. The latter shows the topicality of application of numerical methods to study and predict strength properties of fluid-saturated media under loading.
\end{abstract}

Keywords: porous medium, liquid, fracture, strength

\section{INTRODUCTION}

Porous media, saturated with a fluid, represents a wide class of natural objects, like geological media, including coal seams, oil vessels etc, biological media, such as bone tissue, and artificial ones of different purpose, including filtering materials, endoprostheses etc. [1-3]. At that, the study of the response of such media to mechanical loading, including their deformation and fracture, remains the problem of high importance. In the paper the method of theoretical study of fluid-saturated porous media, namely the hybrid cellular automaton method is proposed, and the results of its application to investigation of features of fracture of brittle specimens, filled with liquid are presented.

\section{BASICS OF HYBRID CELLULAR AUTOMATON METHOD}

Let's consider a medium that consists of a solid skeleton and a system of pores of different scales, filled with liquid. The problem of description of this medium splits into two inter-connected problems: 1) description of mechanical behavior of solid skeleton and 2) description of transfer of fluid in pore volume. In the framework of the proposed approach, we use the movable cellular automaton method [4] to simulate deformation and fracture of a solid skeleton and the finite-difference mesh to simulate a mass transfer of a fluid. These layers, constructed by movable cellular automaton and finite-difference mesh, are interconnected together by means of projection of properties of a solid skeleton from movable cellular automaton to finite-difference mesh and backward projection of

International Conference on Physical Mesomechanics of Multilevel Systems 2014

AIP Conf. Proc. 1623, 115-118 (2014); doi: 10.1063/1.4898896

(C) 2014 AIP Publishing LLC 978-0-7354-1260-6/\$30.00 
pressure of a fluid from finite-difference mesh to movable cellular automaton layer. In the framework of the proposed method a spatial motion and rotation of movable cellular automata leads to transfer of properties (including pressure of a fluid, its concentration etc) of nodes of finite-difference mesh, belonging to corresponding movable cellular automata. This results in the explicit motion of elements of a solid skeleton when modeling the transport of a fluid on finite-difference mesh. The computational accuracy of this approach is determined by the ratio of the size of movable cellular automaton to the size of a cell of finite-difference mesh - the greater this ratio, the more precise the approach. In the framework of movable cellular automaton method (that represents the particles method, formulated as a discrete elements method [5]) we use the principle of multiparticle interaction to describe the forces acting between discrete elements. This approach allows adequate implementation of a variety of rheological models of a solid, including multi-parametric models of plasticity [5]. In the framework of movable cellular automaton method the mechanical response of elastic-plastic material is described within the model of plasticity with non-associated flow law and von Mises yield criterion, also known as Nikolaevsky model [6]. This model postulates the linear dependence between shear strain rate and volumetric strain rate. The mentioned approach allows adequate description of a mechanical response of materials with dilatational plasticity, including the accounting of contributions of underlying scale levels. The Nikolaevsky model of plasticity has been implemented on the basis of Wilkins algorithm that reduces the solution of elastic-plastic problem to the solution on an elastic problem with consequent correction of interaction forces between particles in such a way as to preserve the value of the local pressure constant [7]. An elastic interaction between pair of $i$-th and $j$-th movable automata is defined on the basis of the generalized Hooke's law in hypoelastic form [8]. Mean stress $\bar{\sigma}_{i}^{\text {mean }}$ is calculated on the basis of Love's relation, which states the connection between components of stress tensor $\left(\bar{\sigma}_{\alpha \beta}\right)$, defined in the given volume, and forces, acting on the surface of this volume [4]. In the framework of the developed model it is assumed that the pressure of fluid $P^{\text {fluid }}$ contributes only into the value of hydrostatic stress in solid skeleton (hydrostatic tension). This assumption is adequate under the absence of specific geometry and orientation (texture) of porosity.

The yield stress criterion, which takes into account the contribution of porous pressure of a fluid, is formulated in the following form:

$$
\Phi_{i}=\beta_{i}\left(\bar{\sigma}_{i}^{\text {mean }}+P_{i}^{\text {fluid }}\right)+\bar{\sigma}_{i}^{\text {int }} / \sqrt{3}>Y_{i}
$$

where $Y_{i}$ is shear yield strength of the material of $i$-th element, $\beta_{i}$ is coefficient of internal friction, $\bar{\sigma}_{i}^{\text {int }}$ is von Mises stress (in the volume of $i$-th element). We use the modified Drucker-Prager criterion as the criterion of failure (i.e. loss of the linkage between particles):

$$
\sigma_{\mathrm{DP}}=0.5(a+1) \sigma_{\text {int }}+1.5(a-1)\left(\sigma_{\text {mean }}+P^{\text {fluid }}\right)>\sigma_{\mathrm{c}},
$$

where $\sigma_{\mathrm{c}}$ is the ultimate strength for the given pair of elements, $\sigma_{\mathrm{c}}$ and $\sigma_{\mathrm{t}}$ are compression and tensile strengths of the link between the given pair of elements, $a=\sigma_{\mathrm{c}} / \sigma_{\mathrm{t}}$.

In the framework of the developed model of the liquid transfer we take into account the multi-level structure of porous skeleton by means of introducing of integral parameters of structure from lower scales, namely the value of open porosity (filtration volume), represented by a system of pores and channels in the bulk of material, interconnected with each other and with the surface. We use the following assumptions: 1) a liquid may fill the pore volume as a fully or partially; 2) adsorption of a liquid on surfaces and capillary effects are not taken into account; 3) a liquid is compressible. The equation of transfer of a liquid is based on Leibenzon's equation [9]:

$$
\gamma^{\text {open }} \frac{\partial \rho}{\partial t}=K_{\text {liq }} \nabla\left[\frac{k}{\eta} \nabla \rho\right],
$$

where $K_{\text {liq }}$ is the compression modulus of the liquid, $\rho$ is the density of the liquid, $\gamma^{\text {open }}$ is the open porosity, $\eta$ is the viscosity of the liquid, $\mathrm{k}$ is the coefficient of permeability of solid skeleton, that can be defined as follows: $k=\gamma_{\text {open }} d_{\mathrm{ch}}^{2}$, where $d_{\mathrm{ch}}$ is the characteristic diameter of filtration channels controlling the seepage rate.

As it was told above, in the framework of proposed method a solid skeleton and a liquid are considered as interconnected systems. The influence of pore pressure of a liquid $P_{i}^{\text {fluid }}$ on a movable cellular automaton is 
performed by means of introducing the value of $P_{i}^{\text {fluid }}$ into equations (1), (2). The pore pressure $P_{i}^{\text {fluid }}$ is defined as follows: $P_{i}^{\text {fluid }}=P_{i}^{\text {open }} \gamma_{i}^{\text {open }}$, where $P_{i}^{\text {open }}$ is the pore pressure in open porosity of material of movable automaton.

The mechanical stresses in a solid skeleton lead to changes in volume of open porosity, that in turn, leads to a change of pressure of a fluid. The relation between porosity and mechanical stresses in a solid skeleton is described as follows:

$$
\gamma_{i}^{\text {open }}=\gamma_{\text {init }}^{\text {open }}\left(1+\Omega_{\text {elast }}\right)+\Omega_{\text {plast }},
$$

where $\gamma_{\text {init }}^{\text {open }}$ is the initial value of open porosity, $\Omega_{\text {elast }}$ and $\Omega_{\text {plast }}$ are the local values of elastic and plastic deformations:

$$
\Omega_{\text {elast }}=\frac{3\left(\sigma_{\text {mean }}+P_{i}^{\text {fluid }}\right)}{K}, \Omega_{\text {plast }}=\varepsilon_{x x}+\varepsilon_{y y}+\varepsilon_{z z}-\Omega_{\text {elast }} .
$$

Equations (1)-(5) describe the interference between models of a solid skeleton and a fluid, which are implemented in the framework of the hybrid cellular automaton method.

\section{DEFORMATION AND RESPONSE OF FLUID-SATURATED SAMPLES}

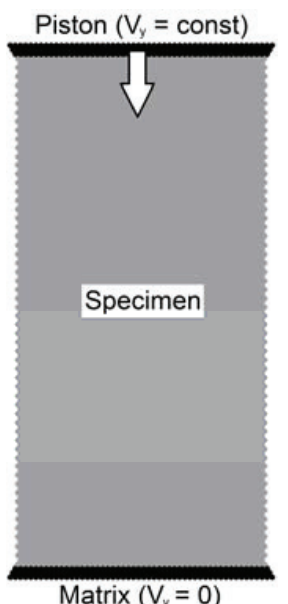

FIGURE 1. The structure of simulated specimen
The developed model was used to simulate the uniaxial loading of porous samples, filled with water. The samples were mounted between immovable supporting plate from below and piston from above (Fig. 1). The piston moved downwards with constant velocity of $V_{y}$. The problem was solved in two-

dimensional formulation in the plane stress approximation. It was assumed that a liquid is absent in the outer space of a specimen. The coefficient of dilatancy of material was $\Lambda=0.173$, the coefficient of internal friction was $\beta=0.49$, the elastic modulus was $K=15 \mathrm{GPa}$, compression and tension strengths were equal, correspondingly, to $\sigma_{\mathrm{c}}=70 \mathrm{MPa}$ and $\sigma_{\mathrm{t}}=10 \mathrm{MPa}$. These properties correspond to the properties of brittle geological material-sandstone. The structure of specimen was assumed to be uniform, without macroscopic pores and inclusions. The peculiarities of the mechanical response of the specimens were studied.

It was revealed that the pore pressure of a liquid plays a significant role for a mechanical response of brittle porous specimens. The fracture in these specimens is realized, as a rule, by means of nucleation and drastic growth of macroscopic crack. During loading, the pore pressure of a liquid in brittle porous specimens increases until fracture of the sample and thus affects its strength and deformation capacity. The dependence of compression strength of specimens of water-saturated porous brittle material on pore pressure is shown in Fig. 2(a).

The compression velocity also significantly influences on the strength of watersaturated specimens (Fig. 2(b)). The generalized dependence of strength on compression velocity and $d_{\text {ch }}$ can be obtained by means of scaling of $x$-axis with the value $d_{\mathrm{ch}} / \sqrt{V_{y} / V_{0}}$, with $V_{0}=1 \mathrm{~m} / \mathrm{s}$. This dependence is described by the following equation:

$$
\sigma_{\mathrm{c}}\left(d_{\mathrm{ch}}, V_{y}\right)=\sigma_{\mathrm{c}}^{0}-\frac{\sigma_{\mathrm{c}}^{\min }-\sigma_{\mathrm{c}}^{0}}{1+\exp \left(\left(d_{\mathrm{ch}}-d_{0}\right) / d_{\mathrm{c}} \sqrt{V_{y} / V_{0}}\right)},
$$

where $\sigma_{0}^{c}$ is the strength of the specimen in the absence of a water in pore volume, $\sigma_{c}^{\min }$ is the strength of watersaturated specimen when a filtration motion of a liquid is forbidden, $d_{0}$ and $d_{\mathrm{c}}$ are parameters, characterizing the position and width of transition zone of the dependence (6). Here $\sigma_{c}^{\min }$ can be estimated as follows: $\sigma_{\mathrm{c}}^{\min }=\sigma_{\mathrm{c}}^{0}\left(1-\gamma^{\text {open }} \frac{a-1}{2} \frac{K_{\text {liq }}}{K}\right)$, that equals to $\sigma_{\mathrm{c}}^{\min }=63.75 \mathrm{MPa}$ for presented calculations and corresponds well to the results, shown in Fig. 2(b). 


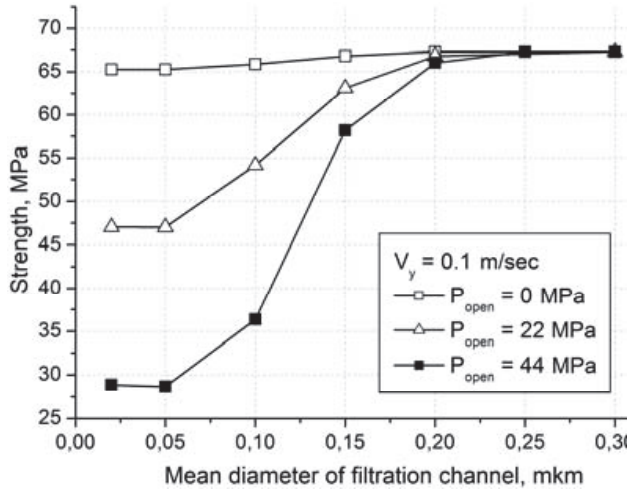

(a)

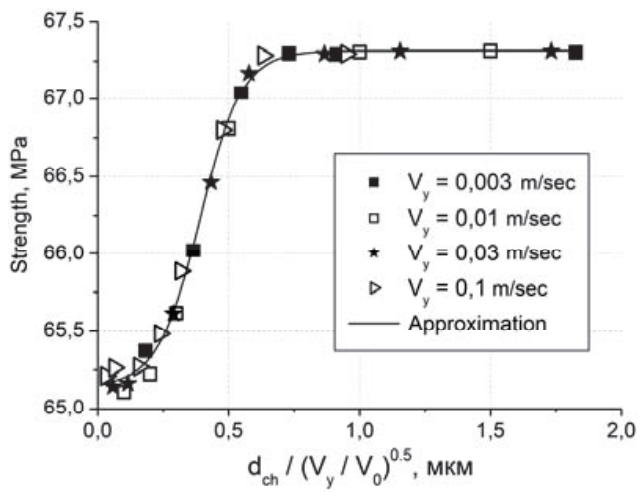

(b)

FIGURE 2. The dependence of compression strength of water-saturated specimens on mean diameter of filtration channel and pore pressure of water (a), the generalized dependence of compression strength of specimens on mean diameter of filtration channel and compression velocity (b)

\section{SUMMARY}

In the paper an approach to simulation of fluid-saturated porous solids is proposed. This approach, called hybrid cellular automaton method combines the movable cellular automaton method with finite-difference method. The proposed approach allows to explicitly take into account both the deformation and fracture of the solid skeleton and mass transfer of the liquid in pore volume of solid.

It was shown that the strength of brittle water-saturated specimens depends on mechanical properties of solid skeleton including permeability, physical-mechanical properties of a liquid, strain rate etc. The generalized dependence of compression strength of specimens on characteristic diameter of filtration channel (and, in other terms, on the permeability of solid) and compression rate was obtained. Deriving analytical expressions for the parameters of this dependence (namely for $d_{\mathrm{c}}, d_{0}, V_{0}$ ) is a task that should be solved in the future.

The study has been carried out within the SB RAS Program III.23.1.4 for Basic Research.

\section{REFERENCES}

1. D. Taylor, J. Mater. Sci. 42, 8911 (2007).

2. Zhejun Pan and L. D. Connell, Int. J. Coal Geol. 69, 243 (2007).

3. S. Zavšek, A. V. Dimaki, A. I. Dmitriev, E. V. Shilko, J. Pezdič, and S. G. Psakhie, Phys. Mesomech. 16(1), 42 (2013).

4. S. Psakhie, E. Shilko, A. Smolin et al., Fract. Struct. Integrity, 24, 59 (2013).

5. S. G. Psakhie, E. V. Shilko, A. Yu. Smolin, A. V. Dimaki, A. I. Dmitriev, Ig. S. Konovalenko, S. V. Astafurov, and S. Zavshek, Phys. Mesomech., 14(5-6), 224 (2011).

6. I. A. Garagash and V. N. Nikolaevskiy, Adv. Mech., 12, 131 (1989).

7. M. L. Wilkins, Computer Simulation of Dynamic Phenomena (Springer-Verlag, Heidelberg, 1999).

8. Yu. P. Stefanov, Phys. Mesomech. 5(5-6), 67 (2002).

9. K. S. Basniev, N. M. Dmitriev, G. V. Chilingar, and M. Gorfunkle, Mechanics of Fluid Flow (John Wiley \& Sons, Inc., Hoboken, 2012). 